\title{
On second order duality for nondifferentiable minimax fractional programming problems involving type-I functions
}

S. K. Gupta ${ }^{1}$ D. Dangar ${ }^{2} \quad$ I. Ahmad $^{3}$

(Received 17 December 2013; revised 11 September 2014)

\begin{abstract}
We introduce second order $(C, \alpha, \rho, d)$ type-I functions and formulate a second order dual model for a nondifferentiable minimax fractional programming problem. The usual duality relations are established under second order $(F, \alpha, \rho, d) /(C, \alpha, \rho, d)$ type-I assumptions. By citing a nontrivial example, it is shown that a second order $(C, \alpha, \rho, d)$ typeI function need not be $(F, \alpha, \rho, d)$ type-I. Several known results are obtained as special cases.
\end{abstract}

http://journal.austms.org.au/ojs/index.php/ANZIAMJ/article/view/7809 gives this article, (C) Austral. Mathematical Soc. 2014. Published November 17, 2014, as part of the Proceedings of the 11th Biennial Engineering Mathematics and Applications Conference. ISSN 1446-8735. (Print two pages per sheet of paper.) Copies of this article must not be made otherwise available on the internet; instead link directly to this URL for this article. 


\section{Contents}

1 Introduction

C480

2 Preliminaries

C481

3 Duality model

C485

References

C492

\section{Introduction}

An optimization problem in which the objective function is the ratio of two functions is a fractional programming problem. It has a wide number of applications in engineering and economics where a ratio of physical or economic functions must be minimised to measure the efficiency or productivity of the system. In mathematical programming, optimization problems in which both a minimization and maximization process is performed are known as minimax (or minmax) problems. Du and Pardalos [5] provided theory, algorithms and applications of some minimax problems. Schmitendorf [13] formulated the following static minimax problem and established necessary optimality conditions:

$$
\text { minimise } f(x)=\sup _{y \in Y} \phi(x, y) \quad \text { subject to } \quad x \in X \subset R^{\mathfrak{n}},
$$

where $\phi: R^{\mathfrak{n}} \times R^{l} \rightarrow R, g: R^{\mathfrak{n}} \rightarrow R^{m}$ are twice continuously differentiable functions, $Y$ is a subset of $R^{l}$ and $X=\left\{x \in R^{n}: g(x) \leqslant 0\right\}$.

Several different minimax fractional programming problems have been studied and duality relations were obtained under various generalized convexity assumptions [3, 7, 8, 9]. Hachimi and Aghezzaf [6] introduced second or$\operatorname{der}(\mathrm{F}, \alpha, \rho, \mathrm{d})$ type-I functions which generalize convexity. Later, Ahmad et al. [2] formulated a second order dual model for a nondifferentiable minimax 
programming problem and proved duality relations under $(F, \alpha, \rho, d)$ type-I functions. Recently, Sharma and Gulati [14] discussed duality results for a minimax fractional programming problem using type-I univex functions.

We first introduce second order ( $C, \alpha, \rho, d)$ type-I functions. A numerical nontrivial example illustrates the existence of such functions. We then formulate a second order dual model involving a vector $r \in R^{n}$ for a nondifferentiable multiobjective fractional programming problem and established weak, strong and strict converse duality theorems under second order $(F, \alpha, \rho, d) /(C, \alpha, \rho, d)$ type-I functions.

\section{Preliminaries}

Throughout this article, gradients and Hessian matrices of the functions $f$, $g, h$ and $\phi$ are with respect to the variable $x$. For instance, $\nabla f(x, y)$ means $\nabla_{x} f(x, y)$. Here, $R^{n}$ denotes the $n$ dimensional Euclidean space, $R_{+}$is the set of nonnegative real numbers and $M=\{1,2, \ldots, \mathfrak{m}\}$.

Definition 1 (Ahmad et al. [2]). A functional $\mathrm{F}: \mathrm{X} \times \mathrm{X} \times \mathrm{R}^{\mathrm{n}} \mapsto \mathrm{R}$, where $\mathrm{X} \subseteq \mathrm{R}^{\mathrm{n}}$, is sublinear with respect to the third variable if for all $(\mathrm{x}, \mathrm{z}) \in \mathrm{X} \times \mathrm{X}$

- $F_{x, z}\left(a_{1}+a_{2}\right) \leqslant F_{x, z}\left(a_{1}\right)+F_{x, z}\left(a_{2}\right)$ for all $a_{1}, a_{2} \in R^{n} ;$ and

- $\mathrm{F}_{x, z}(\alpha \mathrm{a})=\alpha \mathrm{F}_{x, z}(\mathrm{a})$ for all $\alpha \in \mathrm{R}_{+}$and $\mathrm{a} \in \mathrm{R}^{\mathfrak{n}}$.

We now rewrite the definition of second order $(F, \alpha, \rho, d)$ type-I functions introduced by Hachimi and Aghezzaf [6]. Let $F$ be a sublinear functional with respect to the third variable, $\alpha^{1}, \alpha^{2}: X \times X \rightarrow R_{+} \backslash\{0\}, d: X \times X \rightarrow R_{+}$and $\rho^{1}, \rho_{j}^{2} \in R$ for $j \in M$. Let $\phi: X \rightarrow R$ and $g_{j}: X \rightarrow R$ for $j \in M$ be twice differentiable functions.

Definition 2 (Hachimi and Aghezzaf [6]). Function $(\phi, g)$ is second or$\operatorname{der}(\mathrm{F}, \alpha, \rho, \mathrm{d})$ type-I at $z \in \mathrm{X}$ if for all $\mathrm{x} \in \mathrm{X}$ there exists $\mathrm{p} \in \mathrm{R}^{\mathrm{n}}$ such 
that

$$
\begin{aligned}
& \phi(x)-\phi(z)+\frac{1}{2} p^{\top} \nabla^{2} \phi(z) p \geqslant F_{x, z}\left(\alpha^{1}(x, z)\left[\nabla \phi(z)+\nabla^{2} \phi(z) p\right]\right)+\rho^{1} d(x, z), \\
&-g_{j}(z)+\frac{1}{2} p^{\top} \nabla^{2} g_{j}(z) p \geqslant F_{x, z}\left(\alpha^{2}(x, z)\left[\nabla g_{j}(z)+\nabla^{2} g_{j}(z) p\right]\right)+\rho_{j}^{2} d(x, z),
\end{aligned}
$$

for each $\mathbf{j} \in \mathbf{M}$.

Definition 3. Function $(\phi, g)$ is semistrictly second order $(\mathrm{F}, \alpha, \rho, \mathrm{d})$ type-I at $z \in \mathrm{X}$ if for all $\mathrm{x} \in \mathrm{X}$ there exists $\mathrm{p} \in \mathrm{R}^{\mathrm{n}}$ such that

$$
\begin{aligned}
\phi(x)-\phi(z)+\frac{1}{2} p^{\top} \nabla^{2} \phi(z) p & >F_{x, z}\left(\alpha^{1}(x, z)\left[\nabla \phi(z)+\nabla^{2} \phi(z) p\right]\right)+\rho^{1} d(x, z), \\
- & g_{j}(z)+\frac{1}{2} p^{\top} \nabla^{2} g_{j}(z) p \geqslant F_{x, z}\left(\alpha^{2}(x, z)\left[\nabla g_{j}(z)+\nabla^{2} g_{j}(z) p\right]\right)+\rho_{j}^{2} d(x, z),
\end{aligned}
$$

for each $\mathbf{j} \in M$.

Yuan et al. [15] introduced $(\mathrm{C}, \alpha, \rho, \mathrm{d})$ convexity and proved necessary and sufficient optimality conditions for a nondifferentiable multiobjective fractional programming problem. In the framework of this definition, Chinchuluun et al. [4] studied nonsmooth multiobjective fractional programming problems. Later, Long [12] established duality relations for a class of nondifferentiable multiobjective fractional programming problems involving $(C, \alpha, \rho, d)$ convex functions.

We now present $(\mathrm{C}, \alpha, \rho, \mathrm{d})$ type-I functions, after defining convexity in the function $\mathrm{C}$.

Definition 4 (Yuan et al. [15]). A function $\mathrm{C}: \mathrm{X} \times \mathrm{X} \times \mathrm{R}^{\mathrm{n}} \rightarrow \mathrm{R}$ is convex on $\mathrm{R}^{\mathfrak{n}}$ iff for any fixed $(\mathrm{x}, z) \in \mathrm{X} \times \mathrm{X}$ and for any $\mathrm{y}_{1}, \mathrm{y}_{2} \in \mathrm{R}^{\mathfrak{n}}$,

$$
C_{x, z}\left[\lambda y_{1}+(1-\lambda) y_{2}\right] \leqslant \lambda C_{x, z}\left(y_{1}\right)+(1-\lambda) C_{x, z}\left(y_{2}\right),
$$

for all $\lambda \in(0,1)$.

Suppose the real valued function $d: X \times X \rightarrow R_{+}$satisfies $d(x, z)=0$ iff $x=z$ and let $C: X \times X \times R^{n} \rightarrow R$ be a convex function such that $C_{x, z}(0)=0$ for any $(x, z) \in X \times X$. 
Definition 5. Function $(\phi, \mathrm{g})$ is second order $(\mathrm{C}, \alpha, \rho, \mathrm{d})$ type-I at $z \in \mathrm{X}$ if for all $\mathrm{x} \in \mathrm{X}$ there exists $\mathrm{p} \in \mathrm{R}^{\mathrm{n}}$ such that

$$
\begin{aligned}
\frac{1}{\alpha^{1}(x, z)}\left[\phi(x)-\phi(z)+\frac{1}{2} p^{\top} \nabla^{2} \phi(z) p\right] & \geqslant C_{x, z}\left[\nabla \phi(z)+\nabla^{2} \phi(z) p\right]+\frac{\rho^{1} \mathrm{~d}(x, z)}{\alpha^{1}(x, z)} \\
\frac{1}{\alpha^{2}(x, z)}\left[-g_{j}(z)+\frac{1}{2} p^{\top} \nabla^{2} g_{j}(z) p\right] & \geqslant C_{x, z}\left[\nabla g_{j}(z)+\nabla^{2} g_{j}(z) p\right]+\frac{\rho_{j}^{2} d(x, z)}{\alpha^{2}(x, z)}
\end{aligned}
$$

for each $\mathbf{j} \in M$.

Definition 6. Function $(\phi, g)$ is semistrictly second order $(\mathrm{C}, \alpha, \rho, \mathrm{d})$ type-I at $z \in X$ if for all $x \in X$ there exists $p \in R^{\mathfrak{n}}$ such that

$$
\begin{gathered}
\frac{1}{\alpha^{1}(x, z)}\left[\phi(x)-\phi(z)+\frac{1}{2} p^{\top} \nabla^{2} \phi(z) p\right]>C_{x, z}\left[\nabla \phi(z)+\nabla^{2} \phi(z) p\right]+\frac{\rho^{1} \mathrm{~d}(x, z)}{\alpha^{1}(x, z)} \\
\frac{1}{\alpha^{2}(x, z)}\left[-g_{j}(z)+\frac{1}{2} p^{\top} \nabla^{2} g_{j}(z) p\right] \geqslant C_{x, z}\left[\nabla g_{j}(z)+\nabla^{2} g_{j}(z) p\right]+\frac{\rho_{j}^{2} d(x, z)}{\alpha^{2}(x, z)}
\end{gathered}
$$

for each $\mathbf{j} \in M$.

Function $(\phi, g)$ is (semistrictly) second order $(F, \alpha, \rho, d) /(C, \alpha, \rho, d)$ type-I over $X$ iff it is (semistrictly) second order $(F, \alpha, \rho, d) /(C, \alpha, \rho, d)$ type-I at every point in $X$.

Remark 7. If $\mathrm{C}$ is sublinear with respect to the third variable, then Definitions 5 and 6 are identical to Definitions 2 and 3, respectively.

Remark 8. Since the functional $\mathrm{F}$ is sublinear with respect to the third variable, it is convex, as defined in Definition 4. Further, since $\alpha^{1}, \alpha^{2}>0$, every $(F, \alpha, \rho, d)$ type-I function is $(C, \alpha, \rho, d)$ type-I. But the converse need not be true. This is seen from the following example.

Example 9. Let $X=R$. Let $\phi: X \rightarrow R$ and $g: X \rightarrow R$ where $\phi(x)=$ $x^{2}-2 \sin ^{2} x$ and $g(x)=\cos ^{2} x-2 x$. Suppose $\alpha^{1}, \alpha^{2}: X \times X \rightarrow R_{+} \backslash\{0\}$ and $C: X \times X \times R^{n} \rightarrow R$ are $\alpha^{1}(x, z)=1 / 20, \alpha^{2}(x, z)=1 / 3$ and $C_{x, z}(a)=a^{2} / 24$. Let $d: X \times X \rightarrow R_{+}$be $d(x, z)=(x-z)^{2}$. For $p=-1, \rho^{1}=-1 / 20, \rho^{2}=-1$ 
and $z=0.5 \pi$,

$$
\begin{aligned}
& \frac{1}{\alpha^{1}(x, z)}\left[\phi(x)-\phi(z)+\frac{1}{2} p^{\top} \nabla^{2} \phi(z) p\right]-C_{x, z}\left[\nabla \phi(z)+\nabla^{2} \phi(z) p\right]-\frac{\rho^{1} d(x, z)}{\alpha^{1}(x, z)} \\
& =20 x^{2}+40 \cos ^{2} x+60-5 \pi^{2}-\frac{1}{24}(\pi-6)^{2}+(x-0.5 \pi)^{2} \geqslant 0,
\end{aligned}
$$

for all $x \in X$, and

$$
\begin{aligned}
& \frac{1}{\alpha^{2}(x, z)}\left[-g(z)+\frac{1}{2} p^{\top} \nabla^{2} g(z) p\right]-C_{x, z}\left[\nabla g(z)+\nabla^{2} g(z) p\right]-\frac{\rho^{2} d(x, z)}{\alpha^{2}(x, z)} \\
& =\frac{7}{3}+3 \pi+3(x-0.5 \pi)^{2} \geqslant 0,
\end{aligned}
$$

for all $x \in X$. Hence, $(\phi, g)$ is second order $(C, \alpha, \rho, d)$ type-I but $(\phi, g)$ is not second order $(F, \alpha, \rho, d)$ type-I at $z=0.5 \pi$ as $C$ is not sublinear with respect to the third argument.

For $f: R^{n} \times R^{l} \rightarrow R, h: R^{n} \times R^{l} \rightarrow R$ and $g: R^{n} \rightarrow R^{m}$ twice continuously differentiable functions, consider the nondifferentiable minimax fractional programming problem (PP):

$$
\text { minimise } \quad \psi(x)=\sup _{y \in Y} \frac{f(x, y)+\left(x^{\top} B x\right)^{1 / 2}}{h(x, y)-\left(x^{\top} D x\right)^{1 / 2}} \quad \text { subject to } g(x) \leqslant 0,
$$

where $Y$ is a compact subset of $R^{l}, B$ and $D$ are $n \times n$ positive semidefinite matrices, $f(x, y)+\left(x^{\top} B x\right)^{1 / 2} \geqslant 0$ and $h(x, y)-\left(x^{\top} D x\right)^{1 / 2}>0$ for each $(x, y) \in \mathfrak{J} \times Y$, where $\mathfrak{J}=\left\{x \in R^{n}: g(x) \leqslant 0\right\}$. For each $(x, y) \in \mathfrak{J} \times Y$ we define

$$
\begin{aligned}
J(x)= & \left\{j \in M: g_{j}(x)=0\right\}, \\
Y(x)= & \left\{y \in Y: \frac{f(x, y)+\left(x^{\top} B x\right)^{1 / 2}}{h(x, y)-\left(x^{\top} D x\right)^{1 / 2}}=\sup _{z \in Y} \frac{f(x, z)+\left(x^{\top} B x\right)^{1 / 2}}{h(x, z)-\left(x^{\top} D x\right)^{1 / 2}}\right\}, \\
K(x)= & \left\{(s, t, \tilde{y}) \in N \times R_{+}^{s} \times R^{l s}: 1 \leqslant s \leqslant n+1, t=\left(t_{1}, t_{2}, \ldots, t_{s}\right) \in R_{+}^{s},\right. \\
& \left.\sum_{i=1}^{s} t_{i}=1, \tilde{y}=\left(\tilde{y}_{1}, \tilde{y}_{2}, \ldots, \tilde{y}_{s}\right), \tilde{y}_{i} \in Y(x), i=1,2, \ldots, s\right\} .
\end{aligned}
$$




\section{Duality model}

Consider the dual problem (DP) to the PP:

$$
\max _{(s, t, \tilde{y}) \in \mathrm{K}(z)} \sup _{(z, \mu, \lambda, w, v, r, \mathfrak{p}) \in \mathrm{H}_{1}(s, t, \tilde{y})} \lambda,
$$

where $\mathrm{H}_{1}(s, t, \tilde{y})$ denotes the set of all $(z, \mu, \lambda, w, v, r, p) \in R^{n} \times R_{+}^{m} \times R_{+} \times$ $R^{n} \times R^{n} \times R^{n} \times R^{n}$ satisfying

$$
\begin{aligned}
& \sum_{i=1}^{s} t_{i} I\left(z, \tilde{y}_{i}\right)+\nabla^{2} \sum_{i=1}^{s} t_{i}\left[f\left(z, \tilde{y}_{i}\right)-\lambda h\left(z, \tilde{y}_{i}\right)\right] p+\sum_{j=1}^{m} \mu_{j} \nabla g_{j}(z) \\
& +\nabla^{2} \sum_{j=1}^{m} \mu_{j} g_{j}(z) p=0
\end{aligned}
$$

$\sum_{i=1}^{s} t_{i} G\left(z, \tilde{y}_{i}\right)+\left[\sum_{i=1}^{s} t_{i} I\left(z, \tilde{y}_{i}\right)\right]^{\top} r-\frac{1}{2} p^{\top} \nabla^{2} \sum_{i=1}^{s} t_{i}\left[f\left(z, \tilde{y}_{i}\right)-\lambda h\left(z, \tilde{y}_{i}\right)\right] p \geqslant 0$,

$\sum_{j=1}^{m} \mu_{j} g_{j}(z)+\left[\sum_{j=1}^{m} \mu_{j} \nabla g_{j}(z)\right]^{\top} r-\frac{1}{2} p^{\top} \nabla^{2} \sum_{j=1}^{m} \mu_{j} g_{j}(z) p \geqslant 0$,

$\left[\sum_{i=1}^{s} t_{i} I\left(z, \tilde{y}_{i}\right)\right]^{\top} r+\left(\sum_{j=1}^{m} \mu_{j} \nabla g_{j}(z)\right)^{\top} r \leqslant 0$

$w^{\top} \mathrm{B} w \leqslant 1$ and $v^{\top} \mathrm{D} v \leqslant 1$,

where

$$
\begin{aligned}
\mathrm{I}\left(z, \tilde{y}_{i}\right) & =\nabla f\left(z, \tilde{y}_{i}\right)+B w-\lambda\left[\nabla h\left(z, \tilde{y}_{i}\right)-D v\right], \\
\mathrm{G}\left(z, \tilde{y}_{i}\right) & =f\left(z, \tilde{y}_{i}\right)+z^{\top} \mathrm{B} w-\lambda\left[h\left(z, \tilde{y}_{i}\right)-z^{\top} D v\right] .
\end{aligned}
$$

If, for a triplet $(s, t, \tilde{y}) \in K(z)$, the set $\mathrm{H}_{1}(s, t, \tilde{y})=\phi$, then we define the supremum over $\mathrm{H}_{1}$ to be $-\infty$. Now, we establish the duality relations between $\mathrm{PP}$ and DP. 
Theorem 10 (Weak duality). Let $\mathrm{x}$ and $(z, \mu, \lambda, w, v, s, t, \tilde{y}, r, p)$ be feasible solutions of $\mathrm{PP}$ and $\mathrm{DP}$, respectively. Assume that any one of the following four conditions hold:

1. $\left\{G\left(\cdot, \tilde{y}_{i}\right), g_{j}(\cdot), i=1,2, \ldots, s, j=1,2, \ldots, m\right\}$ is second order $(F, \alpha, \rho, d)$ type-I at $z$ and $\sum_{i=1}^{s} t_{i} \rho_{i}^{1}+\sum_{j=1}^{m} \mu_{j} \rho_{j}^{2} \geqslant 0$;

2. $\left\{\sum_{i=1}^{s} t_{i} G\left(\cdot, \tilde{y}_{i}\right), g_{j}(\cdot), j=1,2, \ldots, m\right\}$ is second order $(F, \alpha, \rho, d)$ type-I at $z$ and $\rho^{1}+\sum_{j=1}^{m} \mu_{j} \rho_{j}^{2} \geqslant 0$;

3. $\left\{G\left(\cdot, \tilde{y}_{i}\right), g_{j}(\cdot), i=1,2, \ldots, s, j=1,2, \ldots, m\right\}$ is second order $(C, \alpha, \rho, d)$ type- $I$ at $z$ and $\sum_{i=1}^{s} t_{i} \rho_{i}^{1}+\sum_{j=1}^{m} \mu_{j} \rho_{j}^{2} \geqslant 0$;

4. $\left\{\sum_{i=1}^{s} t_{i} G\left(\cdot, \tilde{y}_{i}\right), g_{j}(\cdot), j=1,2, \ldots, m\right\}$ is second order $(C, \alpha, \rho, d)$ typeI at $z$ and $\rho^{1}+\sum_{j=1}^{m} \mu_{j} \rho_{j}^{2} \geqslant 0$.

Furthermore, suppose $\alpha^{1}(x, z)=\alpha^{2}(x, z)$, then

$$
\sup _{\tilde{y} \in Y} \frac{f(x, \tilde{y})+\left(x^{\top} B x\right)^{1 / 2}}{h(x, \tilde{y})-\left(x^{\top} D x\right)^{1 / 2}} \geqslant \lambda .
$$

Proof: Suppose, contrary to the theorem,

$$
\sup _{\tilde{y} \in Y} \frac{f(x, \tilde{y})+\left(x^{\top} B x\right)^{1 / 2}}{h(x, \tilde{y})-\left(x^{\top} D x\right)^{1 / 2}}<\lambda
$$

then,

$$
f\left(x, \tilde{y}_{i}\right)+\left(x^{\top} B x\right)^{1 / 2}-\lambda\left[h\left(x, \tilde{y}_{i}\right)-\left(x^{\top} D x\right)^{1 / 2}\right]<0,
$$

for all $\tilde{y}_{i} \in Y(x)$ with $i=1,2, \ldots, s$. It follows from $t_{i} \geqslant 0, i=1,2, \ldots, s$, that

$$
t_{i}\left\{f\left(x, \tilde{y}_{i}\right)+\left(x^{\top} B x\right)^{1 / 2}-\lambda\left[h\left(x, \tilde{y}_{i}\right)-\left(x^{\top} D x\right)^{1 / 2}\right]\right\} \leqslant 0,
$$

with at least one strict inequality, since $t=\left(t_{1}, t_{2}, \ldots, t_{s}\right) \neq 0$. Taking the summation over $i$ and using (5),

$$
\sum_{i=1}^{s} t_{i}\left\{f\left(x, \tilde{y}_{i}\right)+x^{\top} B w-\lambda\left[h\left(x, \tilde{y}_{i}\right)-x^{\top} D v\right]\right\}=\sum_{i=1}^{s} t_{i} G\left(x, \tilde{y}_{i}\right)<0
$$


Condition 1: By the second order $(F, \alpha, \rho, d)$ type-I assumption on $\left\{G\left(\cdot, \tilde{y}_{i}\right)\right.$, $\left.g_{j}(\cdot), i=1,2, \ldots, s, j=1,2, \ldots, m\right\}$ at $z$, for $i=1,2, \ldots, s$,

$$
\begin{aligned}
& \mathrm{G}\left(x, \tilde{y}_{i}\right)-\mathrm{G}\left(z, \tilde{y}_{i}\right)+\frac{1}{2} p^{\top} \nabla^{2}\left[f\left(z, \tilde{y}_{i}\right)-\lambda h\left(z, \tilde{y}_{i}\right)\right] p \\
& \geqslant F_{x, z}\left(\alpha^{1}(x, z)\left\{I\left(z, \tilde{y}_{i}\right)+\nabla^{2}\left[f\left(z, \tilde{y}_{i}\right)-\lambda h\left(z, \tilde{y}_{i}\right)\right] p\right\}\right)+\rho_{i}^{1} d(x, z),
\end{aligned}
$$

and, for $\mathbf{j}=1,2, \ldots, \mathrm{m}$,

$$
-g_{j}(z)+\frac{1}{2} p^{\top} \nabla^{2} g_{j}(z) p \geqslant F_{x, z}\left(\alpha^{2}(x, z)\left[\nabla g_{j}(z)+\nabla^{2} g_{j}(z) p\right]\right)+\rho_{j}^{2} d(x, z) .
$$

Multiplying (7) by $t_{i} \geqslant 0, i=1,2, \ldots, s$, multiplying (8) by $\mu_{j} \geqslant 0$, $j=1,2, \ldots, m$, taking summations over $i$ and $j$ and using the sublinearity of $F$, we obtain

$$
\begin{aligned}
& \sum_{i=1}^{s} t_{i} G\left(x, \tilde{y}_{i}\right)-\sum_{i=1}^{s} t_{i} G\left(z, \tilde{y}_{i}\right)+\frac{1}{2} p^{\top} \nabla^{2} \sum_{i=1}^{s} t_{i}\left[f\left(z, \tilde{y}_{i}\right)-\lambda h\left(z, \tilde{y}_{i}\right)\right] p \\
& \geqslant F_{x, z}\left[\alpha^{1}(x, z)\left(\sum_{i=1}^{s} t_{i} I\left(z, \tilde{y}_{i}\right)+\nabla^{2} \sum_{i=1}^{s} t_{i}\left[f\left(z, \tilde{y}_{i}\right)-\lambda h\left(z, \tilde{y}_{i}\right)\right] p\right)\right] \\
& \quad+\sum_{i=1}^{s} t_{i} \rho_{i}^{1} d(x, z), \\
& -\sum_{j=1}^{m} \mu_{j} g_{j}(z)+\frac{1}{2} p^{\top} \nabla^{2} \sum_{j=1}^{m} \mu_{j} g_{j}(z) p \\
& \geqslant F_{x, z}\left[\alpha^{2}(x, z)\left(\sum_{j=1}^{m} \mu_{j} \nabla g_{j}(z)+\nabla^{2} \sum_{j=1}^{m} \mu_{j} g_{j}(z) p\right)\right]+\sum_{j=1}^{m} \mu_{j} \rho_{j}^{2} d(x, z) .
\end{aligned}
$$


Now, using (2), (4) and (6) in (9) and (3) in (10),

$$
\begin{aligned}
& F_{x, z}\left[\alpha^{1}(x, z)\left(\sum_{i=1}^{s} t_{i} \mathrm{I}\left(z, \tilde{y}_{i}\right)+\nabla^{2} \sum_{i=1}^{s} t_{i}\left[f\left(z, \tilde{y}_{i}\right)-\lambda h\left(z, \tilde{y}_{i}\right)\right] p\right)\right] \\
& +\sum_{i=1}^{s} t_{i} \rho_{i}^{1} d(x, z)<-\left[\sum_{j=1}^{m} \mu_{j} \nabla g_{j}(z)\right]^{\top} r
\end{aligned}
$$

and

$$
\begin{aligned}
& F_{x, z}\left[\alpha^{2}(x, z)\left(\sum_{j=1}^{m} \mu_{j} \nabla g_{j}(z)+\nabla^{2} \sum_{j=1}^{m} \mu_{j} g_{j}(z) p\right)\right]+\sum_{j=1}^{m} \mu_{j} \rho_{j}^{2} d(x, z) \\
& \leqslant\left[\sum_{j=1}^{m} \mu_{j} \nabla g_{j}(z)\right]^{\top} r .
\end{aligned}
$$

Finally, using $\alpha^{1}(x, z)=\alpha^{2}(x, z)>0$, in the addition of (11) and (12) and from the sublinearity of $F, \sum_{i=1}^{s} t_{i} \rho_{i}^{1}+\sum_{j=1}^{m} \mu_{j} \rho_{j}^{2} \geqslant 0$ and (1), we have

$$
\begin{aligned}
0=F_{x, z}(0)= & F_{x, z}\left(\sum_{i=1}^{s} t_{i} I\left(z, \tilde{y}_{i}\right)+\nabla^{2} \sum_{i=1}^{s} t_{i}\left[f\left(z, \tilde{y}_{i}\right)-\lambda h\left(z, \tilde{y}_{i}\right)\right] p\right. \\
& \left.+\sum_{j=1}^{m} \mu_{j} \nabla g_{j}(z)+\nabla^{2} \sum_{j=1}^{m} \mu_{j} g_{j}(z) p\right) \\
< & -\left(\sum_{i=1}^{s} t_{i} \rho_{i}^{1}+\sum_{j=1}^{m} \mu_{j} \rho_{j}^{2}\right) \frac{d(x, z)}{\alpha^{1}(x, z)} \leqslant 0,
\end{aligned}
$$

which is a contradiction. Hence the theorem is proved. Similarly, the proof of the theorem can be obtained using Condition 2. 
Condition 3: Since $\left\{G\left(\cdot, \tilde{y}_{i}\right), i=1,2, \ldots, s, j=1,2, \ldots, m\right\}$ is second order $(C, \alpha, \rho, d)$ type-I at $z$, for $i=1,2, \ldots, s$,

$$
\begin{aligned}
& \frac{1}{\alpha^{1}(x, z)}\left\{G\left(x, \tilde{y}_{i}\right)-G\left(z, \tilde{y}_{i}\right)+\frac{1}{2} p^{\top} \nabla^{2}\left[f\left(z, \tilde{y}_{i}\right)-\lambda h\left(z, \tilde{y}_{i}\right)\right] p\right\} \\
& \geqslant C_{x, z}\left(I\left(z, \tilde{y}_{i}\right)+\nabla^{2}\left[f\left(z, \tilde{y}_{i}\right)-\lambda h\left(z, \tilde{y}_{i}\right)\right] p\right)+\frac{\rho_{i}^{1} d(x, z)}{\alpha^{1}(x, z)},
\end{aligned}
$$

and, for $j=1,2, \ldots, m$,

$$
\frac{1}{\alpha^{2}(x, z)}\left[-g_{j}(z)+\frac{1}{2} p^{\top} \nabla^{2} g_{j}(z) p\right] \geqslant C_{x, z}\left[\nabla g_{j}(z)+\nabla^{2} g_{j}(z) p\right]+\frac{\rho_{j}^{2} d(x, z)}{\alpha^{2}(x, z)}
$$

Multiplying (13) by $t_{i} / \tau \geqslant 0$ for $i=1,2, \ldots, s$, and (14) by $\mu_{j} / \tau \geqslant 0$ for $j=1,2, \ldots, m$, where $\tau=1+\sum_{j=1}^{m} \mu_{j}$, we obtain, for $i=1,2, \ldots, s$,

$$
\begin{aligned}
& \frac{1}{\tau \alpha^{1}(x, z)}\left(t_{i}\left\{G\left(x, \tilde{y}_{i}\right)-G\left(z, \tilde{y}_{i}\right)+\frac{1}{2} p^{\top} \nabla^{2}\left[f\left(z, \tilde{y}_{i}\right)-\lambda h\left(z, \tilde{y}_{i}\right)\right] p\right\}\right) \\
& \geqslant \frac{t_{i}}{\tau} C_{x, z}\left(I\left(z, \tilde{y}_{i}\right)+\nabla^{2}\left[f\left(z, \tilde{y}_{i}\right)-\lambda h\left(z, \tilde{y}_{i}\right)\right] p\right)+\frac{t_{i} \rho_{i}^{1} d(x, z)}{\tau \alpha^{1}(x, z)}, \\
& \frac{\mu_{j}}{\tau \alpha^{2}(x, z)}\left[-g_{j}(z)+\frac{1}{2} p^{\top} \nabla^{2} g_{j}(z) p\right] \\
& \geqslant \frac{\mu_{j}}{\tau} C_{x, z}\left[\nabla g_{j}(z)+\nabla^{2} g_{j}(z) p\right]+\frac{\mu_{j} \rho_{j}^{2} d(x, z)}{\tau \alpha^{2}(x, z)} .
\end{aligned}
$$


Summing (15) over $i$ and (16) over $j$, using $\alpha^{1}(x, z)=\alpha^{2}(x, z)$ and the convexity of $\mathrm{C}$,

$$
\begin{aligned}
& \frac{1}{\tau \alpha^{1}(x, z)}\left[\sum_{i=1}^{s} t_{i}\left\{G\left(x, \tilde{y}_{i}\right)-G\left(z, \tilde{y}_{i}\right)+\frac{1}{2} p^{\top} \nabla^{2}\left[f\left(z, \tilde{y}_{i}\right)-\lambda h\left(z, \tilde{y}_{i}\right)\right] p\right\}\right. \\
& \left.-\sum_{j=1}^{m} \mu_{j} g_{j}(z)+\frac{1}{2} p^{\top} \nabla^{2} \sum_{j=1}^{m} \mu_{j} g_{j}(z) p\right] \\
& >C_{x, z}\left[\frac { 1 } { \tau } \left(\sum_{i=1}^{s} t_{i} I\left(z, \tilde{y}_{i}\right)+\nabla^{2} \sum_{i=1}^{s} t_{i}\left[f\left(z, \tilde{y}_{i}\right)-\lambda h\left(z, \tilde{y}_{i}\right)\right] p+\sum_{j=1}^{m} \mu_{j} \nabla g_{j}(z)\right.\right. \\
& \left.\left.+\nabla^{2} \sum_{j=1}^{m} \mu_{j} g_{j}(z) p\right)\right]+\left(\sum_{i=1}^{s} t_{i} \rho_{i}^{1}+\sum_{j=1}^{m} \mu_{j} \rho_{j}^{2}\right) \frac{d(x, z)}{\alpha^{1}(x, z) \tau} .
\end{aligned}
$$

Now, inequalities (2)-(4) yield

$$
\begin{aligned}
& -\sum_{j=1}^{m} \mu_{j} g_{j}(z)+\frac{1}{2} p^{\top} \nabla^{2} \sum_{j=1}^{m} \mu_{j} g_{j}(z) p-\sum_{i=1}^{s} t_{i} G\left(z, \tilde{y}_{i}\right) \\
& +\frac{1}{2} p^{\top} \nabla^{2} \sum_{i=1}^{s} t_{i}\left[f\left(z, \tilde{y}_{i}\right)-\lambda h\left(z, \tilde{y}_{i}\right)\right] p \leqslant 0 .
\end{aligned}
$$

Finally, using (1), (6), (18) and $\sum_{i=1}^{s} t_{i} \rho_{i}^{1}+\sum_{j=1}^{m} \mu_{j} \rho_{j}^{2} \geqslant 0$ in (17),

$$
\begin{aligned}
0=\mathrm{C}_{x, z}(0)=\mathrm{C}_{x, z} & {\left[\frac { 1 } { \tau } \left(\sum_{i=1}^{s} \mathrm{t}_{i} \mathrm{I}\left(z, \tilde{y}_{i}\right)+\nabla^{2} \sum_{i=1}^{s} \mathrm{t}_{i}\left[\mathrm{f}\left(z, \tilde{y}_{i}\right)-\lambda h\left(z, \tilde{y}_{i}\right)\right] p\right.\right.} \\
& \left.\left.+\sum_{j=1}^{m} \mu_{j} \nabla g_{j}(z)+\nabla^{2} \sum_{j=1}^{m} \mu_{j} g_{j}(z) p\right)\right]<0
\end{aligned}
$$

which is a contradiction. Hence the theorem is proved. Similarly, the proof of the theorem can be obtained using Condition 4. 
Theorem 11 (Strong duality). Assume that $x^{*}$ is an optimal solution of $\mathrm{PP}$ and $\nabla \mathrm{g}_{\mathrm{j}}\left(\mathrm{x}^{*}\right)$ for $\mathrm{j} \in \mathrm{J}\left(\mathrm{x}^{*}\right)$ are linearly independent. Then there exist $\left(\mathrm{s}^{*}, \mathrm{t}^{*}, \tilde{\mathrm{y}}^{*}\right) \in \mathrm{K}\left(\mathrm{x}^{*}\right)$ and $\left(\mathrm{x}^{*}, \mu^{*}, \lambda^{*}, w^{*}, v^{*}, \mathrm{r}^{*}=0, \mathrm{p}^{*}=0\right) \in \mathrm{H}_{1}\left(\mathrm{~s}^{*}, \mathrm{t}^{*}, \tilde{\mathrm{y}}^{*}\right)$ such that $\left(\mathrm{x}^{*}, \mu^{*}, \lambda^{*}, w^{*}, v^{*}, \mathrm{~s}^{*}, \mathrm{t}^{*}, \tilde{\mathrm{y}}^{*}, \mathrm{r}^{*}=0, \mathrm{p}^{*}=0\right)$ is a feasible solution of DP and the two objectives have the same values. If, in addition, the assumptions of Theorem 10 hold for all feasible solutions $(x, \mu, \lambda, w, v, s, t, \tilde{y}, r, p)$ of DP, then $\left(x^{*}, \mu^{*}, \lambda^{*}, w^{*}, v^{*}, s^{*}, t^{*}, \tilde{y}^{*}, r^{*}=0, p^{*}=0\right)$ is an optimal solution of DP.

Proof: Since $x^{*}$ is an optimal solution of PP and $\nabla g_{j}\left(x^{*}\right)$ for $j \in J\left(x^{*}\right)$ are linearly independent, then by Theorem 10 and Lai et al. [10] there exist $\left(\mathrm{s}^{*}, \mathrm{t}^{*}, \tilde{\mathrm{y}}^{*}\right) \in \mathrm{K}\left(\mathrm{x}^{*}\right)$ and $\left(\mathrm{x}^{*}, \mu^{*}, \lambda^{*}, w^{*}, v^{*}, \mathrm{r}^{*}=0, \mathrm{p}^{*}=0\right) \in \mathrm{H}_{1}\left(\mathrm{~s}^{*}, \mathrm{t}^{*}, \tilde{\mathrm{y}}^{*}\right)$ such that $\left(x^{*}, \mu^{*}, \lambda^{*}, w^{*}, v^{*}, s^{*}, t^{*}, \tilde{y}^{*}, r^{*}=0, p^{*}=0\right)$ is a feasible solution of DP and the two objectives have same values. Optimality of $\left(x^{*}, \mu^{*}, \lambda^{*}, w^{*}, v^{*}\right.$, $\left.s^{*}, t^{*}, \tilde{y}^{*}, r^{*}=0, p^{*}=0\right)$ for DP thus follows from Theorem 10.

Theorem 12 (Strict Converse Duality). Let $x^{*}$ be an optimal solution of PP and $\left(z^{*}, \mu^{*}, \lambda^{*}, w^{*}, v^{*}, s^{*}, t^{*}, \tilde{y}^{*}, r^{*}, p^{*}\right)$ be an optimal solution of DP. Assume that any one of the following four conditions holds.

1. $\left\{G\left(\cdot, \tilde{y}_{i}^{*}\right), g_{j}(\cdot), i=1,2, \ldots, s^{*}, j=1,2, \ldots, m\right\}$ is semistrictly second $\operatorname{order}(F, \alpha, \rho, d)$ type-I at $z^{*}$ and $\sum_{i=1}^{s^{*}} t_{i}^{*} \rho_{i}^{1}+\sum_{j=1}^{m} \mu_{j}^{*} \rho_{j}^{2} \geqslant 0$.

2. $\left\{\sum_{i=1}^{s^{*}} t_{i}^{*} G\left(\cdot, \tilde{y}_{i}^{*}\right), g_{j}(\cdot), j=1,2, \ldots, m\right\}$ is semistrictly second order $(\mathrm{F}, \alpha, \rho, \mathrm{d})$ type-I at $z^{*}$ and $\rho^{1}+\sum_{j=1}^{m} \mu_{j}^{*} \rho_{j}^{2} \geqslant 0$.

3. $\left\{\mathrm{G}\left(\cdot, \tilde{y}_{i}^{*}\right), g_{j}(\cdot), i=1,2, \ldots, s^{*}, j=1,2, \ldots, m\right\}$ is semistrictly second $\operatorname{order}(\mathrm{C}, \alpha, \rho, d)$ type- $I$ at $z^{*}$ and $\sum_{i=1}^{s^{*}} \mathrm{t}_{i}^{*} \rho_{i}^{1}+\sum_{j=1}^{m} \mu_{j}^{*} \rho_{j}^{2} \geqslant 0$.

4. $\left\{\sum_{\mathfrak{i}=1}^{s^{*}} \mathrm{t}_{i}^{*} \mathrm{G}\left(\cdot, \tilde{\mathrm{y}}_{i}^{*}\right), \mathrm{g}_{\mathfrak{j}}(\cdot), j=1,2, \ldots, \mathrm{m}\right\}$ is semistrictly second order $(\mathrm{C}, \alpha, \rho, d)$ type-I at $z^{*}$ and $\rho^{1}+\sum_{j=1}^{m} \mu_{j}^{*} \rho_{j}^{2} \geqslant 0$. 
Furthermore, suppose the set of vectors $\left\{\nabla \mathrm{g}_{\mathrm{j}}\left(\mathrm{x}^{*}\right), \mathrm{j} \in \mathrm{J}\left(\mathrm{x}^{*}\right)\right\}$ is linearly independent and $\alpha^{1}\left(x^{*}, z^{*}\right)=\alpha^{2}\left(x^{*}, z^{*}\right)$. Then $z^{*}=x^{*}$, that is, $z^{*}$ is an optimal solution of $\mathrm{PP}$.

Proof: The proof follows similarly to the proof of Theorem 10 and Theorem 3.3 of Ahmad et al. [2].

Remark 13. Let B and D be zero matrices of order $\mathfrak{n}$, then the model DP becomes the dual models discussed by $\mathrm{Hu}$ et al. [8]. Further, if $\mathrm{r}=0$, then our dual models reduce to the problems of Husain et al. [7] and Sharma and Gulati [14]. In addition, if $p=0$, then DP becomes the dual model considered by Liu and $\mathrm{Wu}[11]$. If $r=0$ and $p=0$, then the model DP reduces to the model of Ahmad and Husain [1].

\section{References}

[1] Ahmad, I., Husain, Z., Optimality conditions and duality in nondifferentiable minimax fractional programming with generalized convexity. J. Optimiz. Theory Appl. 129:255-275, 2006. doi:10.1007/s10957-006-9057-0 C492

[2] Ahmad, I., Husain, Z., Sharma, S., Second-order duality in nondifferentiable minmax programming involving type-I functions. $J$. Comput. Appl. Math. 215:91-102, 2008. doi:10.1016/j.cam.2007.03.022 C480, C481, C492

[3] Antczak, T., Generalized fractional minimax programming with B-(p,r)-invexity. Comput. Math. Appl. 56:1505-1525, 2008. doi:10.1016/j.camwa.2008.02.039 C480

[4] Chinchuluun, A., Yuan, D. H., Pardalos, P. M., Optimality conditions and duality for nondifferentiable multiobjective fractional programming 
with generalized convexity. Ann. Oper. Res. 154:133-147, 2007. doi:10.1007/s10479-007-0180-6 C482

[5] Du, D.-Z., Pardalos, P. M., Minimax and applications, Kluwer Academic Publishers, Dordrecht, 1995. http://vlsicad.eecs.umich.edu/BK/ Slots/cache/www. wkap.nl/prod/b/0-7923-3615-1 C480

[6] Hachimi, M., Aghezzaf, B., Second order duality in multiobjective programming involving generalized type I functions. Numer. Funct. Anal. Optimiz. 25:725-736, 2005. doi:10.1081/NFA-200045804 C480, C481

[7] Husain, Z., Ahmad, I., Sharma, S., Second order duality for minmax fractional programming. Optimiz. Lett. 3:277-286, 2009. doi:10.1007/s11590-008-0107-4 C480, C492

[8] Hu, Q., Yang, G., Jian, J., On second order duality for minimax fractional programming. Nonlinear Anal. 12:3509-3514, 2011. doi:10.1016/j.nonrwa.2011.06.011 C480, C492

[9] Lai, H. C., Lee, J. C., On duality theorems for a nondifferentiable minimax fractional programming. J. Comput. Appl. Math. 146:115-126, 2002. doi:10.1016/S0377-0427(02)00422-3 C480

[10] Lai, H. C., Liu, J. C., Tanaka, K., Necessary and sufficient conditions for minimax fractional programming. J. Math. Anal. Appl. 230:311-328, 1999. doi:10.1006/jmaa.1998.6204 C491

[11] Liu, J. C., Wu, C. S., On minimax fractional optimality conditions with invexity. J. Math. Anal. Appl. 219:21-35, 1998. doi:10.1006/jmaa.1997.5786 C492

[12] Long, X. J., Optimality conditions and duality for nondifferentiable multiobjective fractional programming problems with (C, $\alpha, \rho, d)$-convexity. J. Optimiz. Theory Appl. 148:197-208, 2011. doi:10.1007/s10957-010-9740-z C482 
[13] Schmitendorf, W. E., Necessary conditions and sufficient conditions for static minmax problems. J. Math. Anal. Appl. 57:683-693, 1977. doi:10.1016/0022-247X(77)90255-4 C480

[14] Sharma, S., Gulati, T. R., Second order duality in minmax fractional programming with generalized univexity. J. Glob. Optimiz. 52:161-169, 2012. doi:10.1007/s10898-011-9694-1 C481, C492

[15] Yuan, D. H., Liu, X. L., Chinchuluun, A., Pardalos, P. M., Nondifferentiable minimax fractional programming problems with (C, $\alpha, \rho, d)$-convexity. J. Optimiz. Theory Appl. 129:185-199, 2006. doi:10.1007/s10957-006-9052-5 C482

\section{Author addresses}

1. S. K. Gupta, Department of Mathematics, Indian Institute of Technology Roorkee, Roorkee 247 667, India mailto:guptashiv@gmail.com

2. D. Dangar, Department of Mathematics and Humanities, IT, Nirma University, Ahmedabad-382481, India mailto:debasisiitp@gmail.com

3. I. Ahmad, Department of Mathematics and Statistics, King Fahd University of Petroleum and Minerals, Dhahran-31261, Saudi Arabia mailto:drizhar@kfupm.edu.sa 